\title{
Analysis on Contact Stress of Disk Insulator
}

\author{
Xinyu Li, Yongzhi Zhang* and Zhuo Li \\ Inner Mongolia University of Technology, Hohhot 010051, China \\ ${ }^{*}$ Corresponding author
}

\begin{abstract}
Disc insulator is one of the most widely used basic components of transmission and distribution system. It is necessary to carry out the stress analysis of its structure. In this paper, ANSYS finite element software is used to simplify the insulator model into an axisymmetric model. The contact stress of the disk insulator is calculated under the ideal condition without considering the material yield, and the stress-strain nephogram is obtained and analyzed.
\end{abstract}

Keywords—disk insulator; stress calculation; Finite Element; axisymmetric; contact analysis

\section{INTRODUCTION}

Disc insulator is a kind of special insulating device, which can play an important role in overhead power transmission line. Usually, a plurality of disc insulators are connected in series to form an insulator string, and the load is mainly axial stretching. Disc insulator mainly by the iron cap, porcelain pieces, cement and steel feet to form a complete system. Insulators in the practical application will be subject to large loads, its safety and practicality is crucial. Therefore, mechanical analysis of the disc insulator is very necessary. To better study the mechanical properties of disc insulators, this paper uses ANSYS finite element analysis software, using the contact algorithm to conduct a Mechanics Analysis of disc insulators.

\section{RELEVANT THEORY OF CONTACT StATE}

This article assumes that the contact body A, the target body $\mathrm{B}$, then from the contact surface is ${ }^{{ }} S_{c}{ }_{c}$, the main contact surface is ${ }^{t} S_{c}^{B}$. On the main contact surface, create a Cartesian coordinate system[1].Then in the local coordinate system, the contact force is expressed as:

$$
{ }^{t} F^{r}={ }^{t} F_{N}^{r t} n^{B}+{ }^{t} F_{1}^{r t} e_{1}+{ }^{t} F_{2}^{r t} e_{2}={ }^{t} F_{N}^{r}+{ }^{t} F_{T}^{r} \quad(r=A, B)
$$

In the local coordinate system, the instantaneous speed is expressed as:

$$
{ }^{t} v^{r}={ }^{t} v_{N}^{r}{ }^{t} n^{B}+{ }^{t} v_{1}^{r t} e_{1}+{ }^{t} v_{2}^{r t} e_{2}={ }^{t} v_{N}^{r}+{ }^{t} v_{T}^{r} \quad(r=A, B)
$$

\section{Contact StRESS ANALYSis MODEL OF DisC INSULATORS}

In this paper, finite element software for static analysis of disc insulators, mainly through four steps, that is, preprocessing, including modeling, setting unit and material parameters, set contact, load and solve, and post-processing. This article chooses a type of disc insulator, let it endure axial $500 \mathrm{KN}$ tensile load. Its structure is mainly composed of four parts, 1 represents the iron cap, 2 and 3 respectively represent the cement a and the cement b, 4 represents ceramic piece, 5 represents steel foot, as shown in Figure 1.

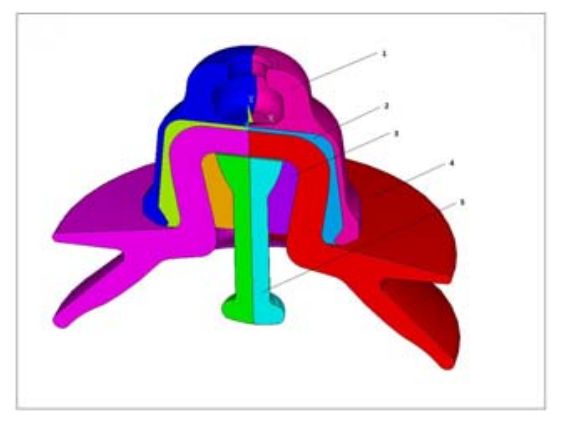

FIGURE I. 1/2 STRUCTURE OF DISC INSULATOR

\section{A. Model Building}

The model of disk insulator can be modeled by the finite element software parameterization, or it can be imported by other software. By removing the partially asymmetrical structure of the iron cap, the disc insulator can be regarded as an axisymmetric structure[2]. Then create a planar twodimensional model, set the axis-related options, which can greatly simplify the model and reduce the computational volume. As shown in Figure 2.

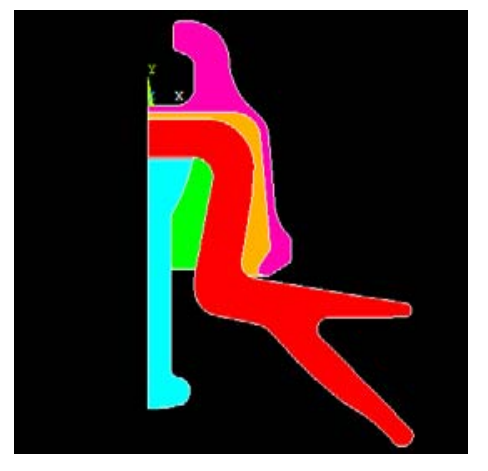

FIGURE II. 2D AXISYMMETRIC MODEL

\section{B. Mesh Generation}

- Select the flat unit type "plane" in the Library of Element Types dialog and add "plane182" unit. Select the Axisymmetric option from the drop-down list in the "Element behavior" field.

- $\quad$ Next, set the material properties, for the model in this paper, only need to define the material's elastic modulus and Poisson's ratio. The material parameters of various components of the disc insulator are shown in Table 1. 
- Next, the four parts of the disk insulator are divided into the grid according to the material properties, the free network mode is selected, and the unit size is selected 2.

\section{TABLE I. MATERIAL PARAMETERS}

\begin{tabular}{|c|c|c|c|c|}
\hline Material & $\begin{array}{c}\text { Iron } \\
\text { cap }\end{array}$ & Cement & $\begin{array}{c}\text { Ceramic } \\
\text { piece }\end{array}$ & $\begin{array}{c}\text { Steel } \\
\text { foot }\end{array}$ \\
\hline $\begin{array}{c}\text { Elastic Modulu } \\
\left(\mathbf{1 0}^{\mathbf{6}} \mathbf{M P a}\right)\end{array}$ & 0.178 & 0.03 & 0.1 & 0.211 \\
\hline Poisson ratio & 0.3 & 0.3 & 0.3 & 0.269 \\
\hline
\end{tabular}

\section{Create Contact Pair}

In this structure, four groups of contact pairs are created. The cemet B and the steel foot are the first group, the porcelain piece and the cement $B$ is the second group, the cement $A$ and the porcelain piece are the third group, the iron cap and the cement A is the fourth group. There are four contact surfaces and four target surfaces. Set friction factor to 0.3. The contact algorithm chooses the penalty function algorithm, and the penalty factor is set to 1.0. Boundary conditions select Standard and Bonded boundaries respectively[3].

\section{Loading and Solving}

- 10 nodes at the head of the iron cap are subjected to 10 constraints, the constraint type is a full DOF constraint.

- The 10 nodes at the bottom of the steel foot are applied with $10 \mathrm{Y}$-axis pulling force, and each node is applied with a pulling force of $50 \mathrm{KN}$.

- Next, the ANSYS finite element software will start nonlinear calculation. When the calculation is finished, the "solution is done"[4] prompt will appear.

\section{STRESS ANALYSIS RESULTS}

When the calculation is finished, this paper analyzes the calculation results and obtains the stress nephogram and the strain nephogram of each component of the disc insulator. This structure adopts axisymmetric model, so the three-dimensional model of disc insulator can be observed by two-dimensional plane graph. Due to the tensile pressure of the disc insulator structure, the analysis of the structure mainly involves the first strength theory, so the stress nephogram of the first main stress is mainly used to analyze it. At the same time, check the strain nephogram to analyze the dangerous deformation.

\section{A. Stress Analysis of the Whole Structure}

According to the stress nephogram of the whole structure, the maximum tensile stress is applied to the axial tension of the steel foot, and the maximum compressive stress is on the cement b.The steel foot is partially tensile stress, and the cement and ceramic piece are mainly compressive stress, and the iron cap is partially pressed partially by the pull, as shown in Figure 3.For the overall strain nephogram, the scale factor is set to 50, and the deformation and undeformed edges are displayed so as to compare the deformation of the structure before and after the tension was loaded. By observing the strain nephogram, the maximum displacement of steel foot can be analyzed, the steel foot and the cement $b$ have been deformed, and the cement $b$ has some degree of compression deformation. The umbrella-shaped parts of the ceramic piece produce the torsional deformation that revolves around the $\mathrm{Z}$ axis. There is little change in the iron cap and the cement. As shown in Figure 4. Then, the local parts are analyzed separately. Cement and ceramic piece are the focus of analysis.

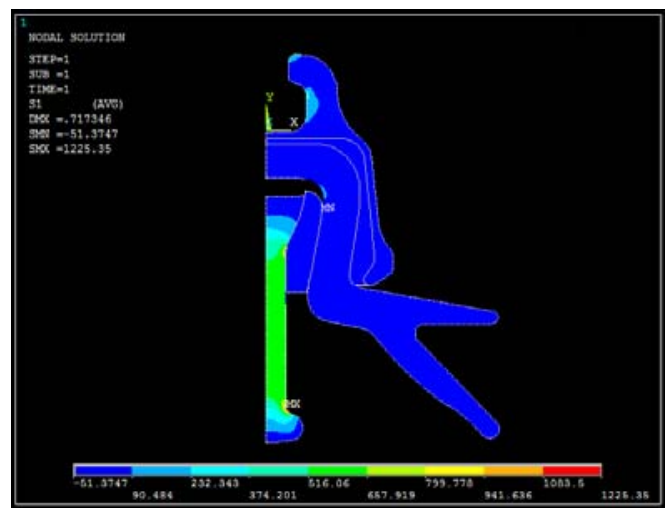

FIGURE III. THE FIRST PRINCIPAL STRESS CONTOUR OF THE WHOLE STRUCTURE

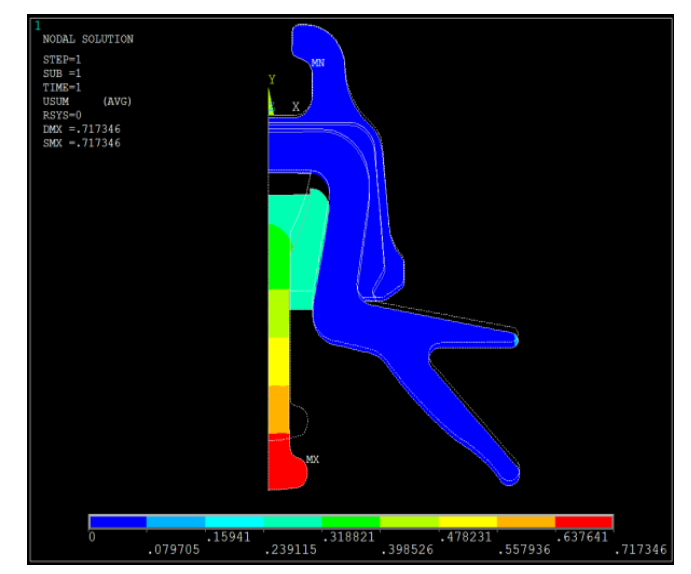

FIGURE IV. THE STRAIN NEPHOGRAM OF THE WHOLE STRUCTURE

\section{B. Stress Analysis of CEMENT}

For the cement, the maximum tensile stress is at the top of the cement a, and the cement a has a stress concentration point at the lower end. This is related to the setting of the contact. The maximum compressive stress is in the cement $b$, the whole cement $\mathrm{b}$ is subjected to compressive stress, and the cement a has tensile stress and compressive stress. As shown in Figure 5.It can be seen clearly in strain nephogram that the cement b has downward displacement and certain compression deformation. The cement a has some downward displacement but it's not obvious. As shown in Figure 6. 


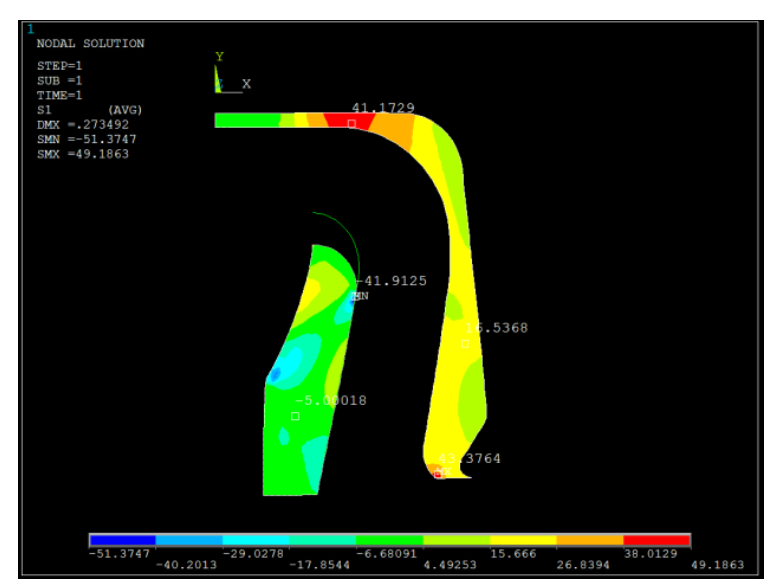

FIGURE V. THE FIRST PRINCIPAL STRESS CONTOUR OF THE CEMENT

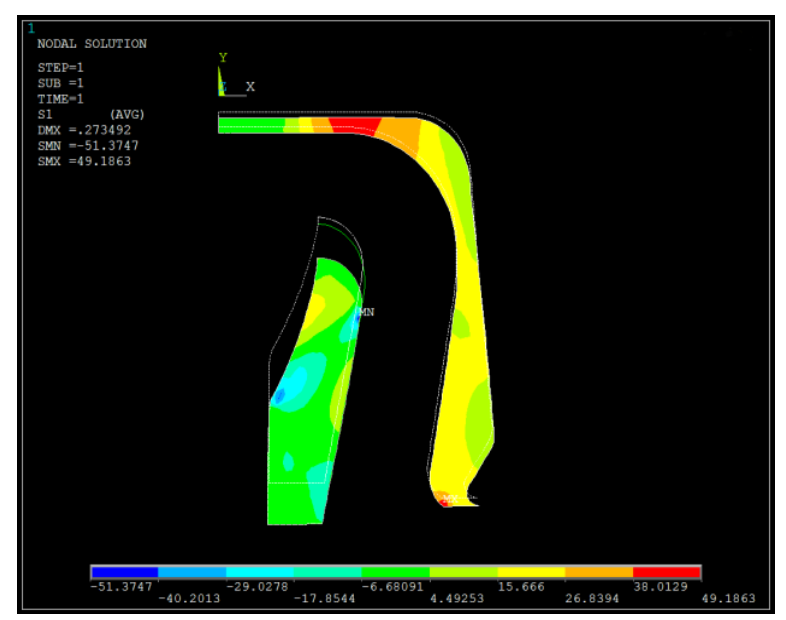

FIGURE VI. THE STRAIN NEPHOGRAM OF THE CEMENT

\section{Stress Analysis of Ceramic Piece}

For the parts of ceramic piece, the maximum tensile stress is between the upper end of the ceramic piece and the umbrella disk, and the maximum compressive stress is in the middle part of the upper end of the ceramic piece. The tensile stress decreases gradually from the maximum tensile stress, and the middle part of the umbrella plate and the upper part of the ceramic piece part is subjected to very small compressive stress, as shown in Figure 7.In the strain nephogram, the maximum displacement is located on the ceramic plate, and the minimum displacement is located on the top of the ceramic piece. However, the overall displacement of the ceramic piece is smaller. At the same time, the umbrella-shaped parts of the ceramic piece produce the torsional deformation that revolves around the $\mathrm{Z}$ axis., but the deformation is smaller, as shown in Figure 7.

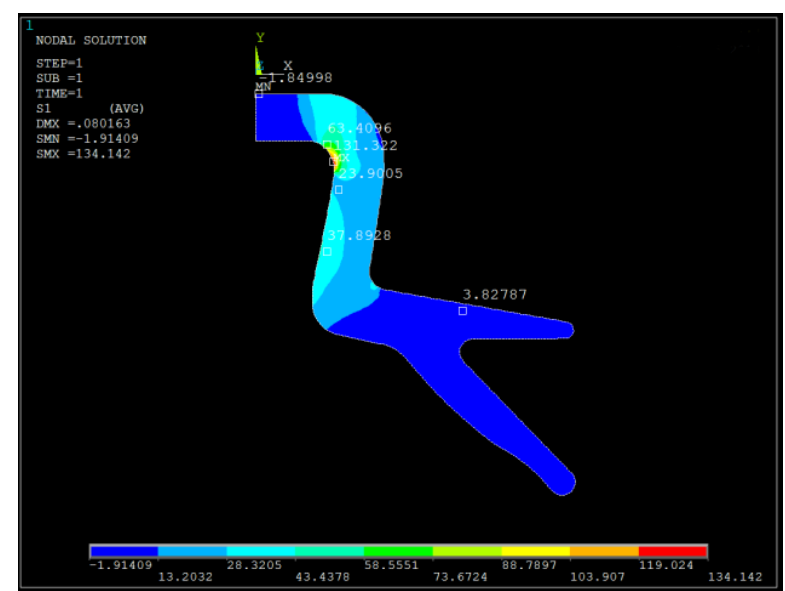

FIGURE VII. THE FIRST PRINCIPAL STRESS CONTOUR OF THE CERAMIC PIECE

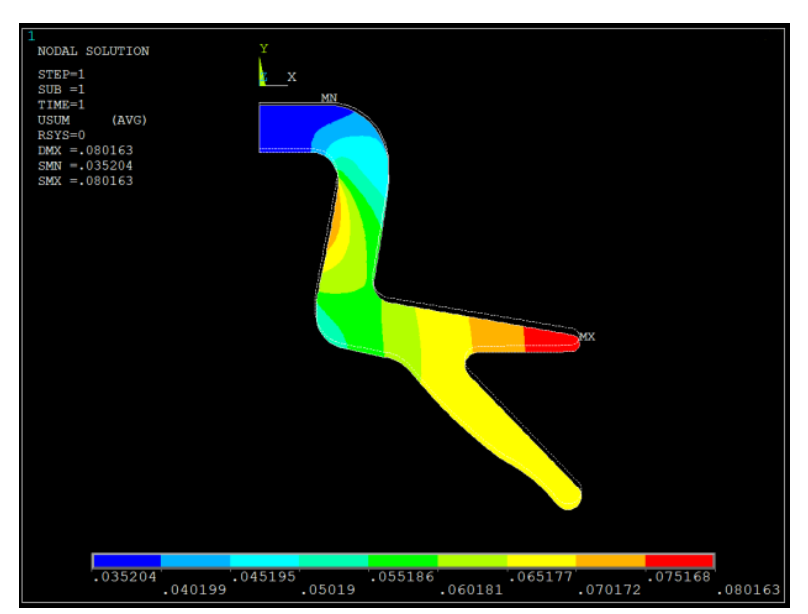

FIGURE VIII. THE STRAIN NEPHOGRAM OF THE CERAMIC PIECE

For the iron cap, the maximum tensile stress is at the bottom of the iron cap at the junction of the cement $a$. The rest is mainly subjected to compressive stress.In the overall strain nephogram, the displacement at the constraint point of the iron cap is 0 , the maximum displacement is in the lower part of the iron cap, and the rest of the displacement is small. For the steel foot, the maximum tensile stress is applied in the area under the steel foot. From this area to the upper end of the steel foot, the tensile stress is gradually reduced. The lower end of the steel foot has a larger downward displacement.

\section{CONCLUSION}

Through the above calculation, according to the stress and strain of each component nephogram, this paper can get some conclusions. For the iron cap, the maximum tensile stress of 467.645MPa, and QT450-10 cast iron tensile strength greater than $450 \mathrm{MPa}$, so generally do not necessarily undermine the iron cap. The maximum tensile stress of the steel foot is 1225.35MPa, while the tensile strength of the 45th steel is $600 \mathrm{MPa}[5]$. It can be seen that the steel foot are easy to be damaged in the stress concentration place. The steel foot with the stress concentrated are about 500MPa, so they will not be damaged. As for the cement, because the cement is generally a cement mixture, it can mainly withstand large compressive 
stress but can not withstand great tensile stress. Tensile strength is generally not more than $10 \mathrm{MPa}$, but the presence of a tensile stress for the cement a $49.1863 \mathrm{MPa}$ area, the tensile stress in this area exceeds the tensile stress limit of the cement, so the part of the cement will be destroyed. The tensile stress in the middle area of the cement $b$ also exceeds the maximum stress limit, so it may also be damaged. For the ceramic piece, the stress cloud shows that the maximum tensile stress is 134.142MPa and the stress in a small area near the maximum stress is also close to $90 \mathrm{MPa}$. Therefore, the tensile stress in this area exceeds the maximum stress limit of ceramic piece, and the ceramic piece may start to be damaged in the area where the maximum tensile stress exists. In summary, the components of the disc insulator structure in this paper are easily damaged parts are cement and ceramic piece, iron cap and steel foot will not be destroyed. Therefore, in this paper, disc insulator should focus on improved design of cement and ceramic piece, or the introduction of more strength materials to ensure the structural safety and stability.

\section{REFERENCES}

[1] Yueguang Wei. A new finite element method for strain gradient theories and applications to fracture analyses[J]. European Journal of Mechanics / A Solids,2006,25(6).

[2] Jun Gao.Structural Optimization Design of Cylindrical Disk Suspension Porcelain Insulator[J].Electromagnetic Absorber,2013(02):24-27.

[3] Zipeng Zhang, Shuqing Wang, Liqin Xue, Xiaohui Yuan. Research on the Diagnosis of Insulator Operating State Based on Improved ANFIS Networks[M].Springer Berlin Heidelberg:2009-06-15.

[4] P. Borkowski,A. Sienicki. Contacts Erosion Modelling Using Ansys Computer Software And Experimental Research[J]. Archives of Metallurgy and Materials,2015,60(2).

[5] Wanyi Tian, Lingyun Yao, Li Li. A Coupled Smoothed Finite ElementBoundary Element Method for Structural-Acoustic Analysis of Shell[J]. Archives of Acoustics,2017,42(1). 